\title{
Provisional study on the 3-D Cauchy condition surface method for fusion plasma shape identification
}

\author{
M. Itagaki ${ }^{1}$, T. Maeda ${ }^{1}$, A. Wakasa ${ }^{1} \&$ K. Watanabe ${ }^{2}$ \\ ${ }^{1}$ Graduate School of Engineering, Hokkaido University, Japan \\ ${ }^{2}$ National Institute for Fusion Science, Japan
}

\begin{abstract}
The 2-dimensional (2-D) Cauchy condition surface (CCS) method to identify the plasma boundary shape has been expanded to deal with 3-D nuclear fusion plasma. This 3-D CCS method solves a set of boundary integral equations in terms of 3-D vector potential with the aid of measured magnetic sensor signals and coil current data. Results obtained in a trial calculation for axisymmetric plasma indicate that this new 3-D method can also determine the plasma boundary shape accurately in the same way as the 2-D CCS method. The authors' research group has a plan to improve this 3-D CCS method to analyse fusion plasma that has a helical geometry.

Keywords: nuclear fusion, plasma boundary, Cauchy condition surface method, magnetic sensor, vector potential, vacuum field, boundary integral equation.
\end{abstract}

\section{Introduction}

To know the boundary shape of plasma in a nuclear fusion device is important for the control of its operation and for diagnostic purposes. As the plasma temperature in such a device is higher than a hundred million deg. K (Kelvin), it is almost impossible to place any sensor inside the plasma. Usually, the plasma boundary shape is indirectly estimated with the aid of on-line computing from signals of magnetic sensors located outside the plasma. For this purpose, the Cauchy condition surface (CCS) method [1] has already been established for a tokamak-type fusion device, e.g., the JT-60 of the Japan Atomic Energy Agency (JAEA). Here, the Cauchy condition surface (CCS) is defined as a surface where 
both the Dirichlet and the Neumann conditions are unknown. The geometry of tokamak plasma is axisymmetric so that the analysis using the CCS method can be made in a 2-dimensional (2-D), r-z system. On the other hand, 3-D analyses are required for non-axisymmtric plasma, e.g., in a helical type device such as the LHD (Large Helical Device) of the National Institute for Fusion Science (NIFS), Japan. The CCS method, however, has not yet been expanded for application to a $3-\mathrm{D}$ space analysis.

The aim of the present work is to investigate whether the CCS method can also be applied to a 3-D system that has a much larger number of unknowns than a 2-D system. Modifying a 3-D boundary element code to solve the Poisson equation, a prototype of 3-D CCS method code has been developed. The present paper deals with tokamak-type axisymmetric plasma, which can be also analyzed using the 2-D CCS method, to demonstrate the fundamental performance as a 3$\mathrm{D}$ code. The authors intend to tackle an actual 3-D geometry such as the helical one in a later paper.

\section{Three-dimensional CCS method}

In the 3-D version of the CCS method proposed here, the CCS $\left(\Gamma_{C}\right)$ is assumed to have a torus shape and to be located in the actual plasma region in 3-D space, as illustrated in fig. 1. In the present work the Dirichlet condition and the Neumann condition along the CCS are the vector potential and its derivative, respectively, while they were the magnetic flux and its derivative in the conventional 2-D CCS method $[1,2]$.

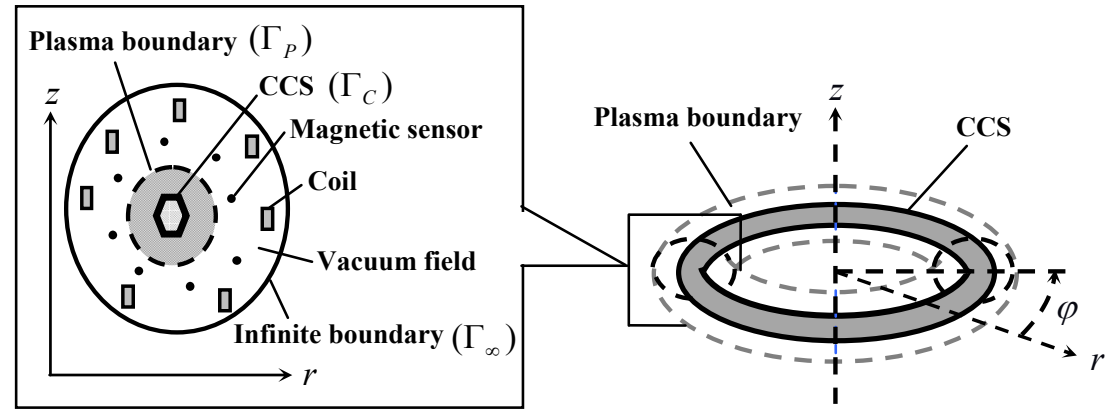

Figure 1: $\quad$ Extention of CCS method to a 3-D system.

The torus shape CCS is divided into a certain number of boundary elements, each of which has 9 nodal points. The first step of the analysis is to obtain the values of the Dirichlet and Neumann conditions at each nodal point in such a way that they will be consistent with the magnetic sensor signals. For this purpose, one solves the set of boundary integral equations for a vacuum field in the same way adopted in the 2-D CCS method calculation. 


\subsection{Vector Laplacian}

The magnetic field $\mathbf{B}$ is given by $\mathbf{B}=\nabla \times \mathbf{A}$ in terms of the vector potential $\boldsymbol{A}$. The relationship between $\boldsymbol{A}$ and the current density $\boldsymbol{j}$ is described by the Poisson equation

$$
\nabla^{2} \boldsymbol{A}=-\mu_{0} \boldsymbol{j} .
$$

In a 3-D Cartesian coordinate system, the two quantities in eqn (1) are expressed as $\boldsymbol{A}=\left(A_{x}, A_{y}, A_{z}\right)$ and $\boldsymbol{j}=\left(j_{x}, j_{y}, j_{z}\right)$. The vector Laplacian in this system has the simple relationship

$$
\left(\nabla^{2} \boldsymbol{A}\right)_{k}=\nabla^{2} A_{k} \quad(k=x, y, z) .
$$

That is, the vector Laplacian can be given by a set of the scalar Laplacian of each Cartesian scalar component. In a cylindrical or spherical coordinate system, on the other hand, the expression of the vector Laplacian is not so straightforward. Because of this, the authors adopt the 3-D Cartesian coordinate system for the analysis to obtain the 3-D distribution of vector potential. However, it is easy to transform the result, once calculated in the Cartesian coordinate system, into the other coordinate systems.

\subsection{Hypothetical assumption of vacuum field}

One here assumes mathematically that there is no plasma current, i.e., vacuum everywhere outside the CCS. The effect of the actual plasma current is transformed into the hypothetical CCS. It is interesting to point out that at any point outside the plasma boundary the vector potential calculated under this assumption is exactly the same as the vector potential caused by the existence of the plasma current. The proof for this is given in the Appendix.

\subsection{Boundary integral equations}

Assuming that the current density term in eqn (1) is the coil current only, the corresponding boundary integral equations for a vacuum field are given by eqns (3) and (4), using the sensor signals and the coil current data.

(i) For points $i$ on the $\operatorname{CCS}\left(\Gamma_{C}\right)$ :

$$
\int_{\Gamma_{C}}\left(\phi_{i}^{*} \frac{\partial A_{k}}{\partial n}-A_{k} \frac{\partial \phi_{i}^{*}}{\partial n}\right) d \Gamma=\frac{1}{2} A_{k, i} . \quad(k=x, y, z) .
$$

(ii) For the magnetic sensor locations $i$ :

$$
\int_{\Gamma_{C}}\left\{\left(\mathcal{L} \phi_{i}^{*}\right) \frac{\partial A_{k}}{\partial n}-A_{k}\left(\mathcal{L} \frac{\partial \phi_{i}^{*}}{\partial n}\right)\right\} d \Gamma=\left(\mathcal{L} A_{k, i}\right)-\mathcal{L} W_{k, i} . \quad(k=x, y, z) .
$$

The fundamental solution $\phi_{i}^{*}$ in eqns (3) and (4) satisfies the 3-D scalar Laplace equation with the Dirac delta function

$$
\nabla^{2} \phi_{i}^{*}=\delta_{i} .
$$


The quantity $W_{k, i}$ in eqn (4) is the contribution of external coil currents to the vector potential at the point $i$, which can be calculated following

$$
\left(W_{x, i}, W_{y, i}, W_{z, i}\right)=\mathbf{W}_{i}=\frac{\mu_{0}}{4 \pi} \sum_{L \in \text { Coils }} \int_{\Omega} \frac{\mathbf{j}_{L}\left(\mathbf{r}_{L}\right)}{\left|\mathbf{r}_{i}-\mathbf{r}_{L}\right|} d \Omega_{L}
$$

with $\mathbf{j}_{L}\left(\mathbf{r}_{L}\right)$ being the electric current density of the L-th coil. Equation (6) can be simplified for an axisymmetric tokamak device. That is, the coil contribution can be represented as a scalar quantity

$$
W_{i}=\mu_{0} \sum_{L \in \text { Coils }} I_{L} \psi^{*}(r, z ; a, b)
$$

using the L-th coil current $I_{L}$ and the fundamental solution of the GradShafranov equation for the axisymmetric geometry $[1,2]$

$$
\psi^{*}(r, z ; a, b)=\frac{\sqrt{a r}}{\pi k}\left[\left(1-\frac{k^{2}}{2}\right) K(k)-E(k)\right]
$$

with

$$
k^{2}=\frac{4 a r}{(r+a)^{2}+(z-b)^{2}} .
$$

The functions $K(k)$ and $E(k)$ are the complete elliptic integrals of the first and second kinds, respectively.

The detailed form of the operator $\mathcal{L}$ in eqn (4) depends on the type of magnetic sensor. In the tokamak-type device, for example, $\mathcal{L}$ is unity for magnetic flux sensors, while for magnetic field sensors it is given by

$$
\mathcal{L}=\sin \theta \frac{1}{r}+\sin \theta \cos \varphi \frac{\partial}{\partial x}+\sin \theta \sin \varphi \frac{\partial}{\partial y}-\cos \theta \frac{\partial}{\partial z},
$$

where $r$ denotes the major radius, $\varphi$ the toroidal angle and $\theta$ the sensor angle respectively, at the sensor position.

Equations (3) and (4) are discretized, coupled and can be expressed in a matrix form. Once all the values of the Dirichlet conditions $\left(A_{k}\right)$ and the Neumann conditions $\left(\partial A_{k} / \partial n\right)$ on $\Gamma_{\mathrm{C}}$ have been given by solving the matrix equation in a least square sense, the distribution of vector potential can be calculated using the boundary integral equation

$$
A_{k, i}=\int_{\Gamma_{C}}\left(\phi_{i}^{*} \frac{\partial A_{k}}{\partial n}-A_{k} \frac{\partial \phi_{i}^{*}}{\partial n}\right) d \Gamma . \quad(k=x, y, z)
$$

for arbitrary points ' $i$ '.

\subsection{Plasma boundary determination}

In a nuclear fusion device, the magnetic field lines lie on nested toroidal magnetic surfaces. A plasma current line also lies on such a magnetic surface. To determine the plasma boundary, it is convenient to introduce a so-called magnetic surface function, $\psi$, which satisfies [3] 


$$
\text { B } \nabla \psi=0 \text {. }
$$

One can define the plasma boundary as the outermost closed surface that is formed with the contour of $\psi$. For example, in tokamak-type axisymmetric plasma, the poloidal magnetic flux function $\psi$ is defined as [1]

$$
\psi=r A_{\varphi},
$$

where $r$ denotes the major radius and $A_{\varphi}$ the toroidal component of the vector potential.

\section{Numerical examples}

The ultimate goal of the authors' research is to identify the shape of nonaxisymmetric plasma. Unfortunately a detailed model for such a complicated system has not yet been completed. As a second best, the case of axisymmetric plasma is dealt with to demonstrate the validity of the present 3-D CCS formulation. The following inverse analysis result is compared with the reference solution given by a direct analysis using the reliable equilibrium code SELENE [4] in JAEA.

One here assumes that 240 magnetic flux sensors and 960 magnetic field sensors are arranged outside the torus shape plasma. The Cauchy condition surface, which also has a torus shape, is placed within a domain that can be supposed to be inside the actual plasma. The ellipse in the centre of fig. 2 is the cross-section of the CCS on the r-z plane. Here the ellipse was set to have a major radius of $0.13 \mathrm{~m}$ and a minor radius of $0.09 \mathrm{~m}$. This 3-D tube-shape CCS was divided into 16 discontinuous quadratic boundary elements, each of which has 9 nodal points.

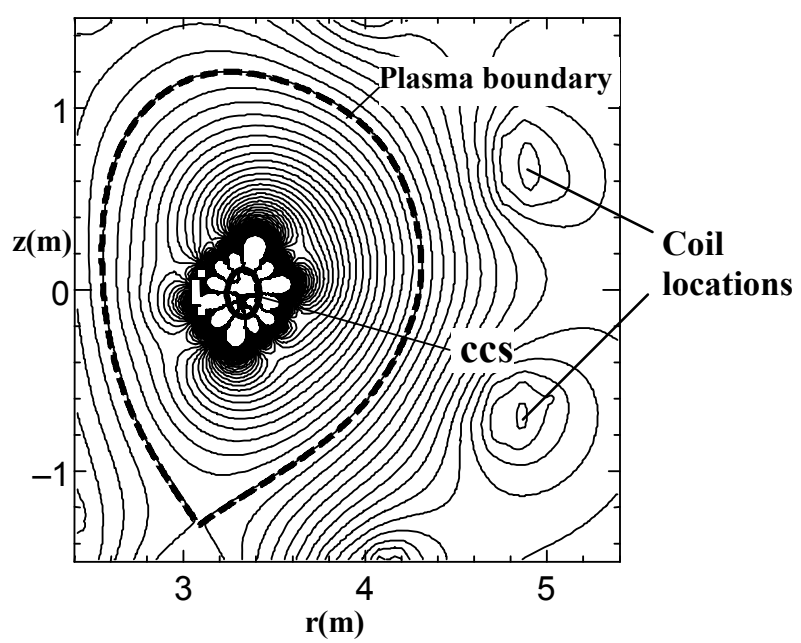

Figure 2: Contours of magnetic flux and the outermost magnetic surface. 
Introducing the external coil current contributions $W_{k, i}$ and the magnetic sensor signals $\mathcal{L} A_{k, i}$, one can calculate $A_{k}$ following eqns (3), (4) and (11). The magnetic flux function $\psi=r A_{\varphi}$ can then be obtained for arbitrary points ' $i$ '. Thus, the outermost magnetic flux surface can be found by drawing contours of $\psi$. The solid lines in fig. 2 are the obtained contours of magnetic flux. Note here that the contours drawn inside the plasma boundary have no physical meaning. Among the contours, however, the outermost closed curve represents the outermost magnetic surface, i.e. the plasma boundary. This reconstructed plasma boundary agrees well with the reference boundary profile (the dotted closed line in fig. 2) that had been assumed beforehand for the SELENE direct analysis. Coil locations outside the plasma are also found in fig. 2 .

\section{Conclusion and further remarks}

A prototype of 3-dimensional CCS method code has been developed, in which the formulation is based on the 3-D distribution of vector potential. Before tackling complicated 3-D geometries, a preliminary 3-D test calculation was made for axisymmetric plasma. The results obtained here indicate that the new 3$\mathrm{D}$ method can also determine the plasma boundary shape accurately in the same way as the 2-D CCS method.

The authors' future plan is to analyse the actual non-axisymmetric 3-D plasma in the LHD, which has a helical geometry. To realise this, the following problems should be solved:

(i) The $\mathrm{x}-$, $\mathrm{y}$ - and z-components in eqn (4) should be solved simultaneously for an actual magnetic field sensor. For a flux loop signal, eqn (4) might be further integrated along the flux loop.

(ii) As it is difficult to derive mathematically the 3-D magnetic surface function $\psi$ that satisfies $\mathbf{B} \cdot \nabla \psi=0$ in a helical system, one needs to seek a practical way of drawing the magnetic field line that is tangential to the field vector.

(iii) Further, the rotational symmetry (not axisymmetry), which is peculiar to LHD, should be incorporated into the boundary integral formulation in order to reduce the number of unknowns.

\section{Acknowledgements}

The authors wish to express their gratitude to Dr. K. Kurihara of JAEA for his helpful guidance on the CCS method. Further, he kindly provided them with the reference JT-60 plasma data used in the numerical demonstration in section 3 . This research was supported by the Ministry of Education, Culture, Sports, Science and Technology, Grant-in-Aid for Scientific Research (C), 20560762, 2008. This work was also performed with the support and under the auspices of the NIFS Collaboration Research Program (NIFS08KLHH308). 


\section{Appendix: validity of the vacuum field assumption for the plasma region}

The proof shown below is written in terms of vector potential. Kurihara originally gave the proof in terms of magnetic flux in his work [5].

Now consider the domain $\Omega_{C-P}$ that is sandwiched between the plasma boundary $\left(\Gamma_{P}\right)$ and the $\operatorname{CCS}\left(\Gamma_{C}\right)$, as illustrated in fig. 3 .

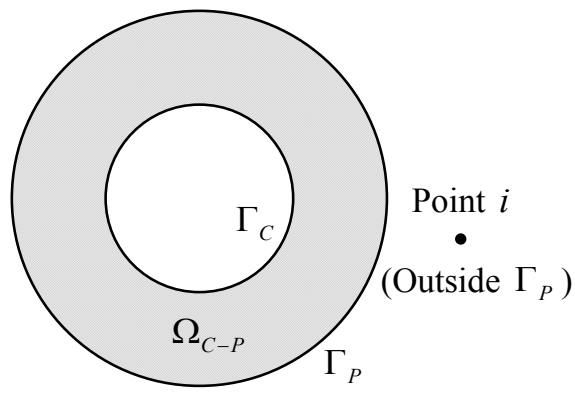

Figure 3: The domain $\Omega_{C-P}$ between $\Gamma_{P}$ and $\Gamma_{C}$.

\section{(i) Vector potential caused by the existence of plasma current:}

The boundary integral equation in this case is written in the form

$$
c_{i} \tilde{A}_{k, i}=\int_{\Gamma_{C}}\left(\phi_{i}^{*} \frac{\partial A_{k}}{\partial n}-A_{k} \frac{\partial \phi_{i}^{*}}{\partial n}\right) \mathrm{d} \Gamma+\int_{\Omega_{C-P}}\left(\mu_{0} j_{k}\right) \phi_{i}^{*} \mathrm{~d} \Omega+W_{k, i} .
$$

By adding

$$
\int_{\Gamma_{P}}\left(\phi_{i}^{*} \frac{\partial A_{k}}{\partial n}-A_{k} \frac{\partial \phi_{i}^{*}}{\partial n}\right) \mathrm{d} \Gamma-\int_{\Gamma_{P}}\left(\phi_{i}^{*} \frac{\partial A_{k}}{\partial n}-A_{k} \frac{\partial \phi_{i}^{*}}{\partial n}\right) \mathrm{d} \Gamma=0
$$

to the RHS of eqn (A1), one obtains

$$
c_{i} \tilde{A}_{k, i}=\int_{\Gamma_{P}}\left(\phi_{i}^{*} \frac{\partial A_{k}}{\partial n}-A_{k} \frac{\partial \phi_{i}^{*}}{\partial n}\right) \mathrm{d} \Gamma+W_{k, i}+c_{i}^{\#} \tilde{A}_{k, i}^{\#},
$$

where the quantity $c_{i}^{\#} \tilde{A}_{k, i}^{\#}$ is given by

$$
c_{i}^{\#} \tilde{A}_{k, i}^{\#}=\int_{\Gamma_{C}-\Gamma_{P}}\left(\phi_{i}^{*} \frac{\partial A_{k}}{\partial n}-A_{k} \frac{\partial \phi_{i}^{*}}{\partial n}\right) \mathrm{d} \Gamma+\int_{\Omega_{C-P}}\left(\mu_{0} j_{k}\right) \phi_{i}^{*} \mathrm{~d} \Omega .
$$

It should be noted that $c_{i}^{\#}=0$ for any point outside $\Omega_{C-P}$. Equation (A1) can then be transformed into

$$
c_{i} \tilde{A}_{k, i}=\int_{\Gamma_{P}}\left(\phi_{i}^{*} \frac{\partial A_{k}}{\partial n}-A_{k} \frac{\partial \phi_{i}^{*}}{\partial n}\right) \mathrm{d} \Gamma+W_{k, i}
$$

that has no term related to the plasma current. 
(ii) Vector potential when assuming a vacuum field for the plasma region:

The CCS method is based on the boundary integral equation for a vacuum field

$$
c_{i} A_{k, i}=\int_{\Gamma_{C}}\left(\phi_{i}^{*} \frac{\partial A_{k}}{\partial n}-A_{k} \frac{\partial \phi_{i}^{*}}{\partial n}\right) \mathrm{d} \Gamma+W_{k, i},
$$

which has no inhomogeneous term related to the plasma current. By adding

$$
\int_{\Gamma_{P}}\left(\phi_{i}^{*} \frac{\partial A_{k}}{\partial n}-A_{k} \frac{\partial \phi_{i}^{*}}{\partial n}\right) \mathrm{d} \Gamma-\int_{\Gamma_{P}}\left(\phi_{i}^{*} \frac{\partial A_{k}}{\partial n}-A_{k} \frac{\partial \phi_{i}^{*}}{\partial n}\right) \mathrm{d} \Gamma=0
$$

to the RHS of eqn (A6), one obtains

$$
c_{i} A_{k, i}=\int_{\Gamma_{P}}\left(\phi_{i}^{*} \frac{\partial A_{k}}{\partial n}-A_{k} \frac{\partial \phi_{i}^{*}}{\partial n}\right) \mathrm{d} \Gamma+W_{k, i}+c_{i}^{\#} A_{k, i}^{\#}
$$

with

$$
c_{i}^{\#} A_{k, i}^{\#}=\int_{\Gamma_{C-P}}\left(\phi_{i}^{*} \frac{\partial A_{k}}{\partial n}-A_{k} \frac{\partial \phi_{i}^{*}}{\partial n}\right) \mathrm{d} \Gamma .
$$

The value of $c_{i}^{\#}$ in eqn (A9) must be zero for any point outside $\Omega_{C-P}$. Thus one obtains

$$
c_{i} A_{k, i}=\int_{\Gamma_{P}}\left(\phi_{i}^{*} \frac{\partial A_{k}}{\partial n}-A_{k} \frac{\partial \phi_{i}^{*}}{\partial n}\right) \mathrm{d} \Gamma+W_{k, i} .
$$

\section{(iii) Conclusion}

As the RHS of eqn (A10) is exactly the same as the RHS of eqn (A5), it is concluded that eqn (A6) is identical to eqn (A1), i.e., $A_{k, i}=\tilde{A}_{k, i}$. That is, the vector potential calculated under the assumption of a vacuum field even for the plasma region is exactly the same as the vector potential caused by the existence of plasma current.

\section{References}

[1] Kurihara, K., Fusion Eng. Des., 51-52, pp. 1049-1057, 2000.

[2] Itagaki, M., Yamaguchi, S., Fukunaga, T., Nucl. Fusion, 45, pp. 153-162, 2005.

[3] Wesson, J, "Tokamaks (Second edition)", The Oxford Engineering Series 48, Clarendon Press, Oxford, 1997.

[4] Takeda, T., Tsunematsu, T., JAERI-M 8042, Japan Atomic Energy Research Institute, 1978.

[5] Kurihara, K., Nucl. Fusion, 33, pp. 399-412, 1993. 